\title{
Esophageal Metastasis to the Iris Effectively Palliated Using Stereotactic Body Radiation Therapy and Adjuvant Intravitreal Chemotherapy: Case Report and Literature Review
}

\author{
Sughosh Dhakal ${ }^{a} \quad$ Gareth M.C. Lema $^{\mathrm{b}}$ \\ David A. DiLoreto $\mathrm{Jr}^{\mathrm{b}}$ Alan W. Katz ${ }^{\mathrm{a}}$ \\ Departments of ${ }^{\mathrm{a}}$ Radiation Oncology and ${ }^{\mathrm{b}}$ Ophthalmology, University of \\ Rochester Medical Center, Rochester, N.Y., USA
}

\section{Key Words}

Esophageal carcinoma - Iris metastasis - Stereotactic body radiation therapy . Bevacizumab - Intravitreal injection

\begin{abstract}
We report a case of isolated iris metastasis from esophageal adenocarcinoma that was successfully managed with local application of stereotactic body radiation therapy (SBRT) and adjunctive intravitreal therapy. A 53-year-old man with locally advanced esophageal adenocarcinoma achieved a complete clinical and radiographic response after surgery and chemotherapy. Four months later, he developed headache and decreased vision and was diagnosed with metastasis to the iris by slit-lamp examination. The decrease in vision was secondary to cystoid macular edema. The metastatic tumor and the patient's symptoms resolved after treatment with SBRT and intravitreal injections of bevacizumab and triamcinolone. We conclude that SBRT combined with intravitreal chemotherapy is an effective and well-tolerated palliative treatment for metastasis of esophageal adenocarcinoma to the iris.
\end{abstract}




\section{Introduction}

Malignant tumors, most often originating in the lung and breast, occasionally metastasize to intraocular structures. Most intraocular metastases occur in the uveal tract (iris, ciliary body, and choroid). In a study of 520 eyes with uveal metastases, only $8 \%$ occurred in the iris among 950 metastatic lesions [1]. In 1 study, the most common complaints were blurred vision, ocular pain, visible iris mass, and photophobia. Visual acuity was $20 / 40$ or better in 27 (68\%) of the 40 affected eyes. There was no history of primary cancer in $32 \%$ of patients [2].

Ocular metastasis is extremely rare in esophageal cancer in general [2], and we are aware of only 1 reported case of metastasis of esophageal cancer to the iris in particular [3], which was treated effectively with systemic chemotherapy. In this case report, we describe an isolated esophageal metastasis to the iris that was effectively palliated using stereotactic body radiation therapy (SBRT).

\section{Case Report}

A 53-year-old man with no significant past medical history presented with a 6-month history of pain when swallowing and regurgitation. Esophagogastroduodenoscopy revealed a large, circumferential, distal esophageal mass, with biopsy revealing adenocarcinoma. Staging PET/CT did not reveal any regional lymphadenopathy or distant spread of disease. The patient underwent an en bloc esophagectomy, with pathology demonstrating T3N2 (stage IIIB) disease, and he was subsequently treated with adjuvant chemotherapy. He was well, with no clinical or radiographic evidence of disease recurrence for 4 months, until he began experiencing pain and vision loss in his left eye.

Ophthalmic exam showed a visual acuity of 20/20 in the right eye and 20/100 in the left. Intraocular pressure in both eyes and an examination of the right eye was normal. In the left eye, the pupil was irregular and poorly reactive, but there was no afferent pupillary defect. There was a cystic

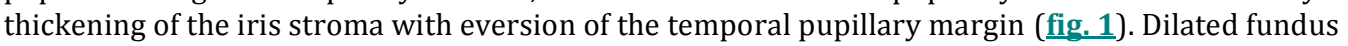
exam of the left eye showed cystoid macular edema, but no choroidal metastasis. Ultrasound biomicroscopy and anterior segment OCT suggested that the lesion was only in the iris and did not involve the ciliary body, although it may have extended to the iris root. Contrast-enhanced CT of the head and MRI of the brain and orbit did not reveal any abnormal mass or suspicious enhancing lesions. In addition, a restaging PET/CT scan did not reveal disease recurrence elsewhere in the body.

The patient was diagnosed clinically with isolated metastasis to the iris. Intraocular biopsy was not performed to avoid the risk of tumor seeding at distant sites via the iris vascularization. He began treatment with intravitreal bevacizumab $1.25 \mathrm{mg}$ for cystoid macular edema and was referred for radiation therapy. The patient was simulated in the supine position and a frameless technique was utilized, which included a custom aquaplast mask with U-frame for immobilization. The previously acquired contrast-enhanced CT and MRI were fused to the planning CT simulation, and all previous clinical evaluations were carefully analyzed to help delineate the target volume and normal surrounding critical structures. The gross tumor volume was defined, and then the clinical target volume was determined. Due to concerns about eye motion, the clinical target volume was contoured to include as much of the anterior half of the orbit as possible. A planning target volume margin of 2 mm was added.

The Novalis ExacTrac patient positioning platform (Brainlab AG, Heimstetten, Germany) was used for immobilization. Treatment planning was performed using the BrainScan system (Brainlab AG). Highly conformal radiation therapy, consisting of 8 fixed beams, was used to deliver 35 Gy in fourteen 2.5-Gy daily fractions using $6 \mathrm{MV}$ photons with a 0.5 - $\mathrm{cm}$ bolus (fig. 2 ). For quality assurance, CT fusions were done after the dry run and fifth fraction. 
The patient received a total of 2 bevacizumab injections separated by 1 month. The second occurred 3 days before his first radiation treatment. When the patient returned 3 weeks later, he had acute anterior uveitis with $3+$ anterior chamber cell and flare. His vision had decreased to 20/400, and cystoid macular edema worsened. Rather than bevacizumab, $4 \mathrm{mg}$ of intravitreal Triesence was administered. One month later, which was 2 weeks after completion of SBRT, the patient reported subjective visual improvement. Anterior uveitis and cystoid macular edema were improving. Treatment continued with prednisolone acetate $1 \%$ four times daily in the left eye. One month later, the iris metastasis had totally regressed and his vision improved to 20/150. At follow-up 6 months after the last radiation treatment, there was no recurrence of the iris metastasis (fig. 1), his macular edema had totally resolved, and Snellen visual acuity had returned to 20/30.

\section{Discussion}

Intraocular structures are uncommon sites of distant metastasis. Metastasis to the choroid is most common, while involvement of the iris is rare [1]. Complications of iris metastasis include decrease in visual acuity and secondary glaucoma. The goal of treatment is alleviation of clinical symptoms and preservation of visual acuity, while survival outcomes are dictated by the prognosis of the primary disease. Treatment options include chemotherapy or radiotherapy, while resistant cases may require enucleation for relief of severe symptoms.

A number of reports have documented effective local control of ocular metastasis with conventional fractionated radiotherapy. The majority of the studies used doses between 30 and 40 Gy with 2-3 Gy fractions over a 3-4 week period, with response rates up to $89 \%$ [4-6]. Brachytherapy using intraocular plaques has also been used [1], providing the advantage of shorter treatment duration but requiring an invasive procedure. SBRT is a non-invasive treatment that can be administered in a much shorter time period than traditional fractionated external beam radiation therapy. Although its efficacy and safety profile have been established in nearly every primary malignancy and disease site throughout the body, we are aware of only 1 report detailing its use in metastasis to the eye. Bellmann et al. [7] reported on 10 patients with unifocal choroidal metastasis who were treated using SBRT with 12-20 Gy in a single fraction or $30 \mathrm{~Gy}$ in 10 fractions. With a median follow-up period of 6.5 months, local control was achieved in all treated lesions, with 5 lesions showing radiographic evidence of tumor regression. All but 3 patients had stable or improved visual symptoms.

Intravitreal bevacizumab has been used as an adjuvant therapy for choroidal metastases from various primary tumors and has caused tumor regression in metastases that either failed chemotherapy or developed months after primary chemotherapy was completed $[8,9]$. The indications for intravitreal bevacizumab were loss of vision or threatened loss of central vision. Most eyes had lasting recovery of vision.

In this case, our patient received 2 bevacizumab injections without significant shortterm visual improvement. However, since he received SBRT simultaneously, we cannot speculate on the effects of bevacizumab as a primary treatment. The use of bevacizumab is a reasonable option for management of ocular metastases, especially if other effects of the metastases are seen, e.g. macular edema. 


\section{Acknowledgment}

The authors thank Ms. Laura Finger for editorial assistance.

\section{Disclosure Statement}

The authors declare no conflicts of interest
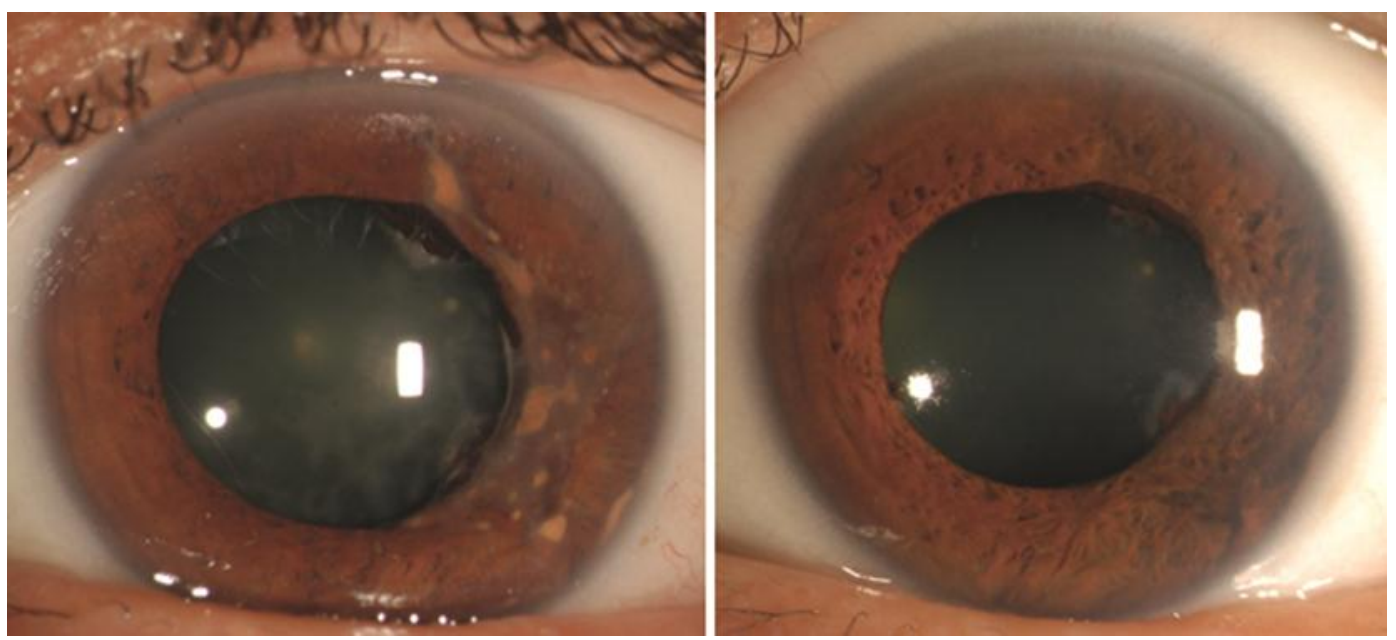

Fig. 1. Iris mass, 2 weeks after the first injection of bevacizumab (left) and 6 months after external beam radiation (right). The mass has resolved, but posterior synechiae and ectropion uvea persist. 


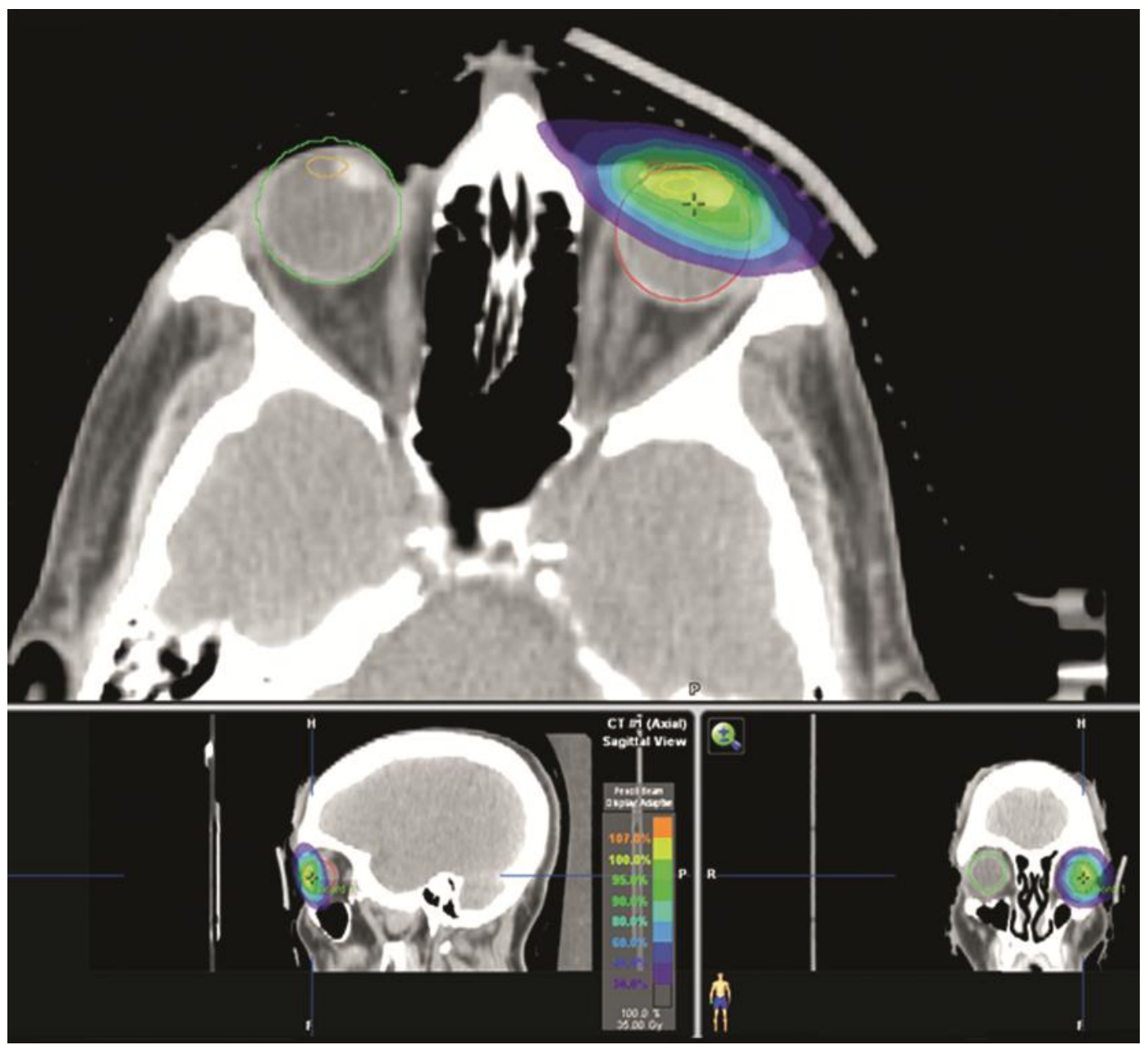

Fig. 2. Isodose distribution in axial, coronal, and sagittal planes.

\section{References}

1 Shields CL, Shields JA, Gross NE, et al: Survey of 520 eyes with uveal metastases. Ophthalmology 1997;104:1265-1276.

2 Shields JA, Shields CL, Kirati H, et al: Metastatic tumors to the iris in 40 patients. Am J Ophthalmol 1995;119:422-430.

3 Ichiki Y, Morita M, Yano K, et al: Iris metastasis of esophageal cancer. Ann Thorac Surg 2005;79:1782-1784.

4 Wiegel T, Bornfeld N, Kreusel EM, et al: Radiotherapy for choroidal metastases: interim analysis of a prospective study of the ARO (AR095-08). Front Radiat Ther Oncol 1997;30:154-159.

5 Rudoler SB, Corn BW, Shields CL, et al: External beam irradiation for choroid metastases: identification of factors predisposing to long-term sequelae. Int J Radiat Oncol Biol Phys 1997;38:251-256.

6 Brady LW, Shields JA, Augsburger JJ, et al: Malignant intraocular tumors. Cancer 1982;49:578-585.

7 Bellmann C, Fuss M, Holz FG, et al: Stereotactic radiation therapy for malignant choroidal tumors: preliminary, short-term results. Ophthalmology. 2000;107:358-365.

8 Kim SW, Kim M, Huh K, et al: Complete regression of choroidal metastasis secondary to non-small-cell lung cancer with intravitreal bevacizumab and oral erlotinib combination therapy. Ophthalmologica 2009;223:411413.

9 Sanchez de la Barquera Cordero A, Hidalgo R: Intravitreal bevacizumab for choroidal metastasis of lung carcinoma; case report. J Ophthalmic Vis Res 2010;5:265-268. 